\title{
Effect of Illumination Time on Water Treatment Efficiency of Symbiotic Bacteria and Algae
}

\author{
Zhenwei Wang, Wei Zuo*, Jun Zhang, Congcong Tang \\ School of Municipal and Environmental Engineering, Harbin Institute of Technology, Harbin, China \\ Email address: \\ wzwstyle@126.com (Zhenwei Wang), zuoweistar@163.com (Wei Zuo) \\ ${ }^{*}$ Corresponding author
}

\section{To cite this article:}

Zhenwei Wang, Wei Zuo, Jun Zhang, Congcong Tang. Effect Of Illumination Time On Water Treatment Efficiency Of Symbiotic Bacteria And Algae. Science Discovery. Vol. 5, No. 4, 2017, pp. 301-306. doi: 10.11648/j.sd.20170504.20

Received: May 23, 2017; Accepted: June 8, 2017; Published: June 27, 2017

\begin{abstract}
Using SBR to study the influence of light time on the effluent quality of the symbiotic system of symbiotic bacteria and algae, so as to get the appropriate operating parameters. In the artificial sewage treatment as the goal, then mixed with two sink pool algae and sewage treatment plant activated sludge system, start algae inter-growth, when HRT was 9h, and the temperature is $(25 \pm 1)^{\circ} \mathrm{C}$ and $\mathrm{pH}$ is about 7 , the same water and other parameters are fixed. The change of illumination time, examine the effluent quality and sludge properties, so as to obtain the sewage treatment effect of optimized illumination time. The results showed that when the illumination time was 8 hours, the removal efficiency of total nitrogen, total phosphorus and COD was best, and the activity of SBR was better. Considering the light time is 8 hours, the dark state of the domestic sewage treatment is better for 4 hours.
\end{abstract}

Keywords: Algal-Bacterial Symbionts, SBR, Illumination Time

\section{光照时间对菌藻共生系统水处理效能的影响的研究}

王振威, 左薇", 张军, 唐聪聪

市政环境工程学院, 哈尔滨工业大学, 哈尔滨市, 中国

邮箱

wZwstyle@126.com(王振威), zuoweistar@163.com(左薇)

摘要：本文主要研究了光照时间的变化对菌藻共生SBR系统处理污水效能的影响。以人工配制的生活污水为目标处理 物, 将二沉池池壁藻类和污水处理厂活性污泥进行混合, 利用获得的菌藻共生系统对生活污水进行处理。在HRT为9h、 温度为 $(25 \pm 1)^{\circ} \mathrm{C} 、 \mathrm{pH}$ 为7左右、光强为4000lux、进水相同等其他参数不变的情况下, 研究不同的光照时间对污水处理 效能的影响, 通过考察出水的水质以及污泥性质, 从而获得最为适宜的光照时间。实验结果表明, 光照时间对COD和 氨氮的去除效果最为明显，当光照时间为 $8 \mathrm{~h}$ 时，去除效果显著提高，COD的去除率为 $91.19 \%$, 氨氮的去除率为 $91.07 \%$ 。 同时, 光照时间为 8 小时, 菌藻SBR对总氮、总磷以及COD的去除效果均较好, SVI为 70.8 , 污泥活性和沉降性均较为 良好。

关键词：菌藻共生, SBR, 光照时间 


\section{1. 引言}

菌藻共生系统有节约曝气、降低能耗、回收资源、深 度脱氮除磷等多种优点, 是当今备受关注的新型绿色污水 深度处理技术 $[1]$ 。Su等人对比研究了席藻、衣藻、小球藻 和栅藻4种蓝绿藻的污水处理效能, 发现以小球藻为代表 的绿藻具有最佳的氮磷去除效果, 无机氮去除率接近 $100 \%$, TP去除率为 $96.9 \%$ 以上 [2]; Van DenHende[3]等人 研究无机碳与有机碳比例对藻菌共生系统氮磷处理效能 的影响时, 发现绿藻-细菌共生系统可实现氮磷的深度处 理，其处理效率可达 $80 \%$ 。

Veena[4]等人对菌藻之间的相互作用类型进行了深入 的研究, 其研究结果表明, 菌藻共生系统表现在两个方面, 即协同作用与抑制作用。协同作用是菌藻相互影响的增效 作用。藻类对城市生活污水中的氮、磷等营养元素的去除 以及对有机物的摄取具有一定的功效, 将其与细菌降解污 染物的能力有效地结合在一起。藻类能够进行光合作用并 释放一定量的氧气, 将其供给好氧异养微生物, 从而进行 生产代谢活动等; 而好氧异养微生物也可以通过对有机污 染物质的氧化分解供给藻类在光合作用中所需的碳源和 营养物质, 如二氧化碳、磷化合物以及无机氮等代谢产物, 如此循环往复, 便形成了菌藻之间互利共生的关系。并以 此实现了微生物有效生长和出水水质优化的良性循环。抑 制作用即为菌藻之间的相互竞争。在这种竞争的影响下, 菌、藻系统的处理效能会出现彼此削弱的现象。菌藻之间 相互争夺营养物质, 并且可以通过释放抑制剂以及毒素影 响另一方的生长, 甚至将其致死。当这种抑制作用占据主 导时, 菌藻系统不仅无法发挥各自的功能, 去除水中污染 物质, 同时可能因为抑制作用使得微生物细胞和胞外物质 死亡, 甚至导致水体愈加恶化, 出水水质变差等。

近些年来, 为了考察菌藻系统的氮磷处理效果, 中国 和国外学者从菌藻共生系统的优势藻类篮选、菌藻相互 作用机制、系统操作条件优化等方面入手进行了深入研究 [5]。现如今所研究的菌藻共生系统依附的反应器多数为氧 化塘, 但水力停留时间普遍较长。而SBR工艺是现如今一 种较为成熟且高效的污水处理工艺, 在很多情况下都有所 使用。而且, 在大多数环境下 (包括工业废水在内的污水 处理), 不需要设置调节池[6]。序批式活性污泥法的一大 特点就是污泥容易沉降, 污泥容积指数低[7]。因此, 轻易 不会产生污泥膨胀等不利于污水处理效能的现象; 如若采 取适当的运维管理方式, 污水处理效能更佳, 且耐冲击负 荷能力较强, 出水水质也普遍优于连续式水处理工艺 [8]。 Valigore等利用SBR培养菌藻混合污泥, 在HRT为 $1.4 \mathrm{~d}$ 时, 其COD的处理效率可达 $77 \%$ [9]。 Tang等人在研究中发现, 当HRT缩短至原有时长的 $1 / 10$ 时, 系统中藻类的量浓度由 $0.726 \mathrm{~g} / \mathrm{L}$ 降至 $0.061 \mathrm{~g} / \mathrm{L}$, 藻类大量流失量, 导致氮磷去除 效率的显著下降[10]。

为解决菌藻共生系统面临的瓶颈。提高菌藻共生系统 水处理效率并减缓藻类流失, 沿用SBR工艺。SBR工艺使 用范围很广, 适合多种不同条件的应用, 也是一种高效且 成熟的污水处理工艺。将藻类引入SBR反应器中, 使活性 污泥与藻类协同处理污水, 能够弥补SBR 在脱氮除磷方面 的不足, 具有同步脱氮除磷、资源回收、节能降耗等技术
优势, 是城镇污水处理引人关注的发展方向, 具有研究意 义缩短HRT, 将水力停留时间设置为 12 小时。据此研究光 照时间等其他因素对菌藻共生系统的影响, 以期实现菌藻 共生系统在高效污水处理工艺中的稳定生长。

\section{2. 实验部分}

\section{1. 篮选藻种}

根据文献内容, 菌藻共生系统适宜选取小球藻。根据 实际情况, 选取污水处理厂二沉池中池壁的藻类为实验藻 种, 采集少许, 并进行离心浓缩。弃去上清液后, 剩余物 质便可用于微藻的富集培养。将经过浓缩处理后的藻类放 入投加营养物质的烧杯内并持续摚拌, 采用LED灯模拟自 然条件, 保持黑暗时间与光照时间 $1: 1$ 的比例, 每 $4 \mathrm{~d}$ 换1次 水, 并密切观察藻类的生长情况, 最终获得用于本次实验 的藻类。于显微镜下观察富集培养后的藻类, 发现主要为 栅藻和小球藻。如图1所示。

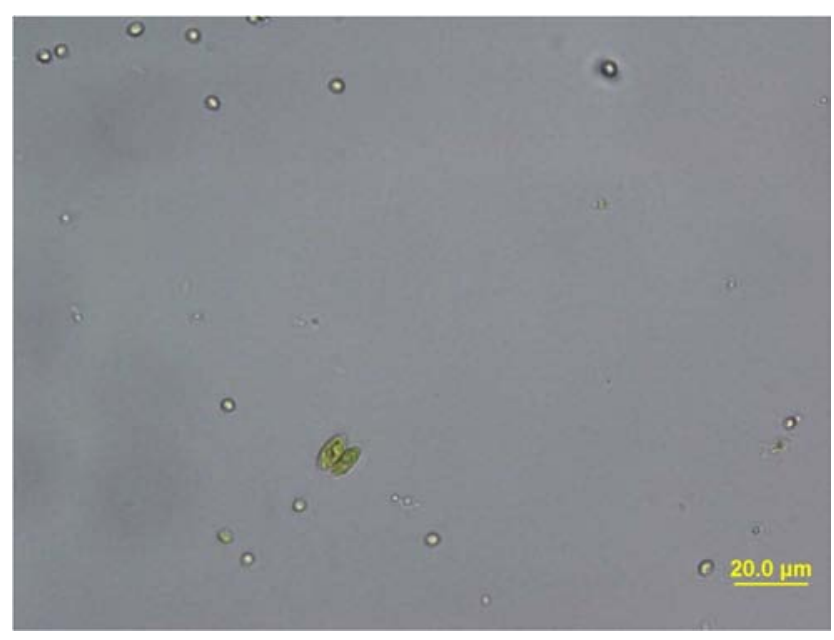

图1 镜检观察到的小球藻和栅藻。

\section{2. 实验仪器}

主要装置和仪器为: 分析天平、离心机、马弗炉、紫 外分光光度计、溶解氧测定仪、烘箱、 $\mathrm{pH}$ 计等。具体装置 及仪器见表1。

表1 装置及仪器。

\begin{tabular}{ll}
\hline 电子天平 & 北京赛多利斯仪器系统有限公司 \\
\hline TDL-5-A离心机 & 上海安亭科学仪器厂 \\
磁力加热搅拌器 & 金坛市双捷实验仪器厂 \\
ET3150B多功能消解器 & 上海欧陆科仪有限公司 \\
紫外分光光度计 & 北京普析通用仪器有限公司 \\
ET1151M型COD测定仪 & 上海欧陆科仪有限公司 \\
光学显微镜 & 日本多美公司 \\
pH仪 & 梅特勒-托利多仪器 (上海) 有限公司 \\
溶解氧测定仪 & 厦门隆力德环境技术开发有限公司 \\
SX2-4-10马弗炉 & 上海嘉展仪器设备有限公司 \\
电热恒温鼓风干燥箱 & 上海跃进医疗器械厂 \\
JA21001型电子天平 & 上海精密科学仪器有限公司 \\
LZB-3WB转子流量计 & 常州市科德热工仪表有限公司 \\
立式压力蒸汽灭菌器 & 上海博讯实业有限公司医疗设备厂 \\
震荡器 & 海门市其林贝尔仪器制造有限公司 \\
\hline
\end{tabular}




\section{3. 配制模拟生活污水}

在本次实验中，菌藻SBR的进水均为实验室配制的生 活污水。具体方法如下: 在 $25 \mathrm{~L}$ 水中加入药品并充分搅拌, 使其混匀。各组分分别为: 葡萄糖 $6.0 \mathrm{~g}$ 、淀粉 $6.0 \mathrm{~g}$ 、碳酸 氢钠 $14.0 \mathrm{~g}$ 、硫酸镁 $0.3 \mathrm{~g}$ 、磷酸二氢钾 $1.0 \mathrm{~g}$ 、氯化铵 $3.0 \mathrm{~g}$ 、 氯化钙 $0.1 \mathrm{~g}$ 。具体水质组成见表 2 。

表2 实验室配制生活污水组成。

\begin{tabular}{ll}
\hline 药品名称 & 质量分数 $(\%)$ \\
\hline 葡萄糖 & 0.24 \\
可溶性淀粉 & 0.24 \\
碳酸氢钠 & 0.3 \\
磷酸二氢钾 & 0.048 \\
硫酸镁 & 0.04 \\
无水氯化钲 & 0.004 \\
氯化铵 & 0.152 \\
\hline
\end{tabular}

本次实验所用的污泥来源于某污水处理厂的活性污 泥。实验前进行活化十天。

\section{4. 实验方法}

使用 4 个 $2 \mathrm{~L}$ 的烧杯作为反应器, 分别标号 $1 \#-4 \#$ 。反 应器的形式为菌藻共生 SBR。SBR每天运行 2 个周期, 每 次运行十二小时。其中, 进水 1 小时, 反应 9 小时, 静置 1 小时, 排水 1 小时, 换水率为 $60 \%$, 反应器的 $\mathrm{pH}$ 应保持在 7 左右, 溶解氧的质量浓度维持在 $5 \mathrm{mg} / \mathrm{L}$ 左右。1\#-4\#反应 器每个反应周期的光照时间分别为 $6 、 8 、 10 、 12$ 小时。经 过测量, 接种的菌藻生物量为 $2000 \mathrm{mg} / \mathrm{L}$, 每天排泥 $100 \mathrm{ml}$, 藻类的接种体积与活性污泥的接种体积比为 $1: 4$ 。

\section{5. 测试方法}

具体测试方法见表 3 。

表3 水质指标测定方法。

\begin{tabular}{ll}
\hline 水质指标 & 检测及分析方法 \\
\hline 氨氮 & 纳氏试剂分光光度法 \\
总磷 & 钼酸铵分光光度法 \\
正磷酸盐 & 钼酸铵分光光度法 \\
亚硝态氮 & N- ( 1 -菱基 $)$-乙二胺光度法 \\
硝态氮 & 酚二磺酸光度法 \\
$\mathrm{COD}$ & 重铬酸钾法 \\
总氮 & 碱性过硫酸钾消解-紫外分光光度法 \\
叶绿素 & 丙酮分光光度计法 \\
$\mathrm{pH}$ & pH计 \\
$\mathrm{DO}$ & 便携式溶解氧测定仪 \\
\hline
\end{tabular}

\section{3. 结果与讨论}

\section{1. 光照时间对出水 COD的影响}

如图2所示, 四个反应器对 COD的平均去除率分别为 $86.67 \% 、 91.19 \% 、 88.46 \% 、 87.31 \%$ 。且随着反应天数的 增加, 1 号反应器逐渐出现了波动。其中, 2 号反应器对 COD 的去除效果最好, 而且十分稳定, 波动较小。

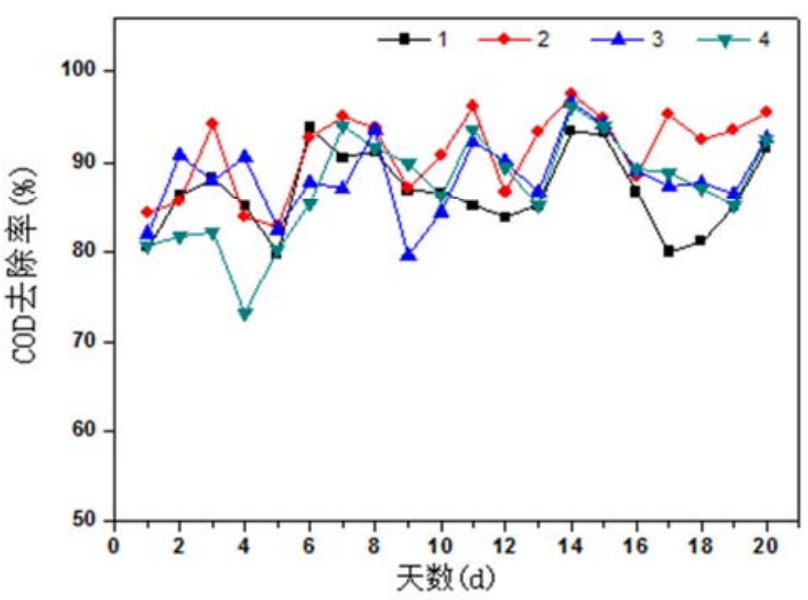

图2 不同光照时间运行条件下COD的去除情况。

从图中可以看出, 2 号、 3 号、 4 号反应器随着光照时 间的不断延长, 除效果逐渐变差, 这可能是由于形成了光 抑制, 从而影响了藻类正常的代谢。所以, 菌藻SBR系统 需要适宜的光照时间。

\section{2. 光照时间对出水氮类的影响}

\subsection{1. 光照时间对出水氨氮的影响}

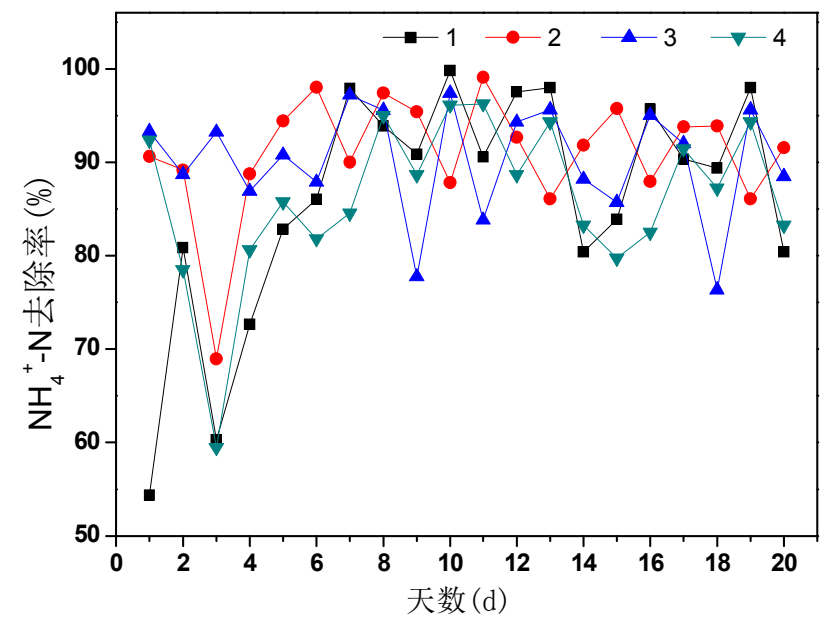

图3 不同光照时间运行条件下氨氮的去除情况。

四个反应器对氨氮的去除效果如图 3 所示, 除了 3 号反应器, 其他三组反应器在前期均存在一定的波动, 2 号反应器大约 5 天之后逐渐稳定, 而 4 号反应器始终存在较 大的波动。四组反应器对氨氮的平均去除率分别为 $86.18 \%$ 、 $90.18 \% 、 91.07 \% 、 87.53 \%$, 其中 2 号和 3 号反应器的去除 效果较好。在反应后期, 3 号反应器波动较 2 号稍大。

\subsection{2. 光照时间对出水硝态氮的影响}

硝氮的浓度变化如图4所示。其中反应器进水的硝态 氮平均浓度为 $1.53 \mathrm{mg} / \mathrm{L}$ 。在反应初期, 四组反应器硝态氮 的浓度均较低, 从第十天开始, 出现了较为明显的波动。 四组反应器出水中硝氮的浓度分别为 $6.98 \mathrm{mg} / \mathrm{L} 、 4.24 \mathrm{mg} / \mathrm{L}$ 、 $5.71 \mathrm{mg} / \mathrm{L}$ 和 $6.03 \mathrm{mg} / \mathrm{L}$ 。 1 号反应器在整个反应周期内均存 在较大的波动, 出水中硝态氮的浓度十分不稳定。而 2 号 
和 3 号反应器除了在第十二天和第十八天出现了较大的波 动外, 出水中硝态氮的浓度都比较稳定, 均在 $10 \mathrm{mg} / \mathrm{L}$ 以下。 可以认为, 四组反应器虽然偶有波动, 但相差不大, 还需 考量其他氮类指标以及总氮的去除效果。

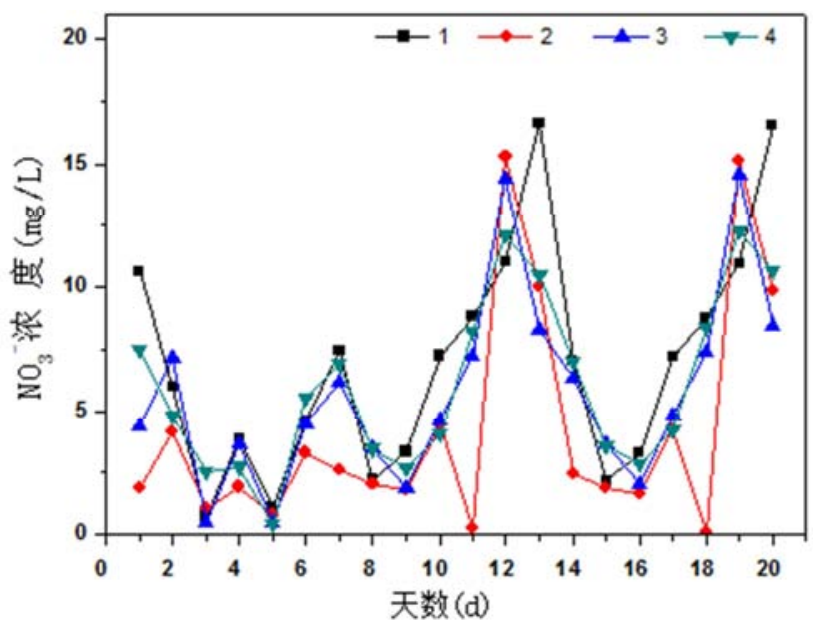

图4 不同光照时间运行条件下硝态氮的浓度变化。

\subsection{3. 光照时间对出水亚硝态氮的影响}

图5显示了整个反应周期内出水水质中亚硝氮的浓度 变化。其中, 四组反应器中进水的亚硝态氮的浓度为 $0.06 \mathrm{mg} / \mathrm{L}$ 。而四组反应器出水中亚硝态氮的平均质量浓度 分别是 $0.13689 \mathrm{mg} / \mathrm{L} 、 0.12168 \mathrm{mg} / \mathrm{L} 、 0.68445 \mathrm{mg} / \mathrm{L}$ 、 $0.39546 \mathrm{mg} / \mathrm{L}$ 。其中 2 号和 3 号反应器出水中亚硝态氮浓度 平稳, 没有出现大的波动, 均在 $1 \mathrm{mg} / \mathrm{L}$ 以下, 而 1 号和 4 号 反应器却在第 3 天和第 11 天出现了较大的波动。

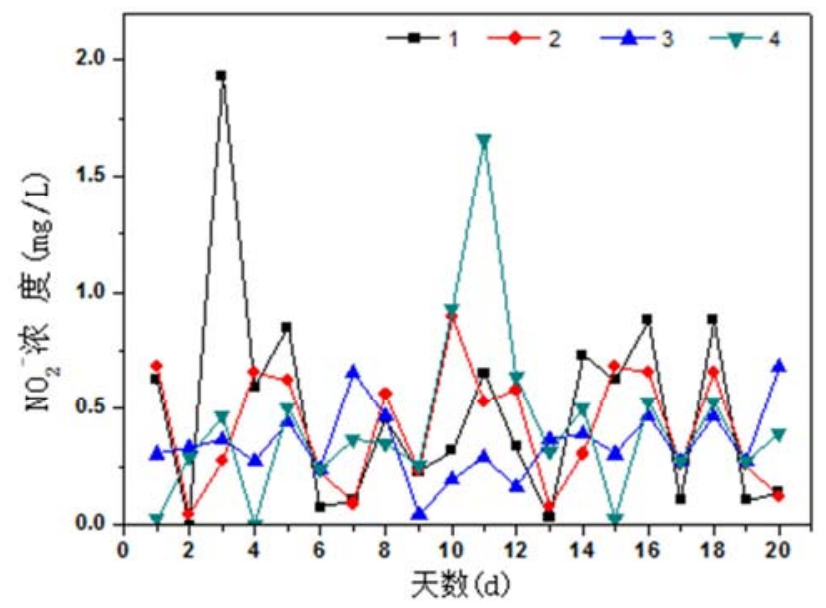

图5 不同光照时间运行条件下亚硝态氮的浓度变化。

\subsection{4. 光照时间对出水总氮的影响}

不同光照时间对出水水质中TN的影响如图6所示。1 号反应器和 4 号反应器前期波动较大, 一周后才逐渐稳定, 且 1 号反应器对 TN的去除效果最差。1 号反应器去除效果 差是因为光照时间较短, 藻类无法获取充足的光能, 对氮 类物质的转化受到抑制。而且 1 号和 4 号反应器前期波动较 大, 后期的去除效果才逐渐平稳, 可见光照时间也影响了
菌藻共生SBR的启动。其他两组反应器在运行期间始终较 为稳定只存在较小的波动。四个反应器的平均去除率分别 为 $84.22 \% 、 89.72 \% 、 88.76 \% 、 84.61 \%$ 。由图可知， 2 号反 应器和 3 号反应器的的出水中TN的去除率较好, 且都始终 维持在较高的水平。而且, 2 号反应器的光照时间更短, 有利于节约能源。

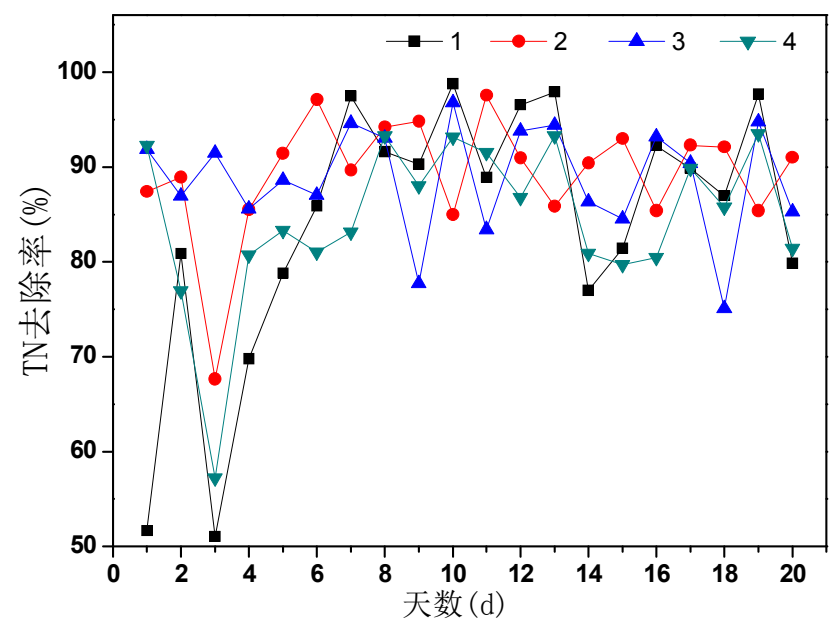

图6 不同光照时间运行条件下总氮的去除情况。

\section{3. 光照时间对出水中磷的影响}

\section{3. 1. 光照时间对出水中正磷酸根的影响}

光照时间对出水正磷酸盐的去除效果如图7所示, 4 组反应器的平均去除率为 $65.98 \%$ 、 $80.23 \%$ 、 $74.96 \%$ 和 $67.61 \%$ 。由此可知, 2 号菌藻反应器的去除效果明显优于 其他三组, 这说明其他光照时间 8 个小时, 黑暗 4 个小时, 最适宜菌藻的共生。能够最大化的发挥菌藻共生的作用。 而且, 1 号和 4 号反应器的处理效果最差, 而且在整个反 应周期内, 1 号反应器和 4 号反应器存在较大的波动。这 也表明光照时间过长或者过短, 均会影响菌藻系统的处 理效能。

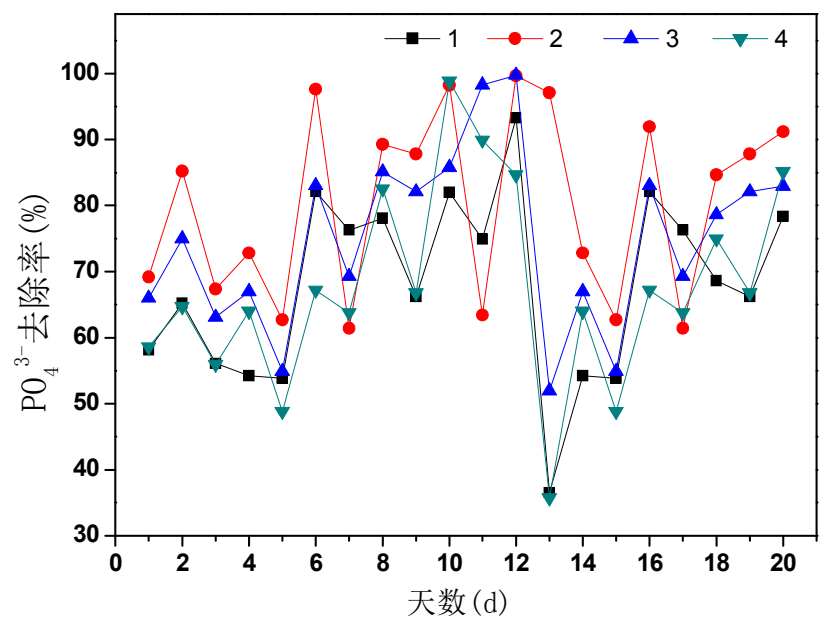

图7 不同光照时间运行条件下正磷酸根的去除情况。 


\subsection{2. 光照时间对出水中总磷的影响}

光照时间对出水总磷的去除效果如图 8 所示, 4组反应 器的平均去除率为 $61.35 \% 、 78.61 \% 、 72.16 \%$ 和 $67.08 \%$ 。 可以明显看出, 总磷的去除效果与正磷酸根的去除效果基 本一致。由图可知, 2 号菌藻反应器的去除效果明显优于 其他三组, 这说明其处于黑暗 4 个小时、光照时间为 8 个小 时, 最适宜菌藻的共生, 并且能够最大化的发挥菌藻共生 的作用。而 1 号和 4 号反应器的处理效果最差, 尤其是在中 后期, 处理效果明显弱于 2 号和 3 号。且存在较大的波动。

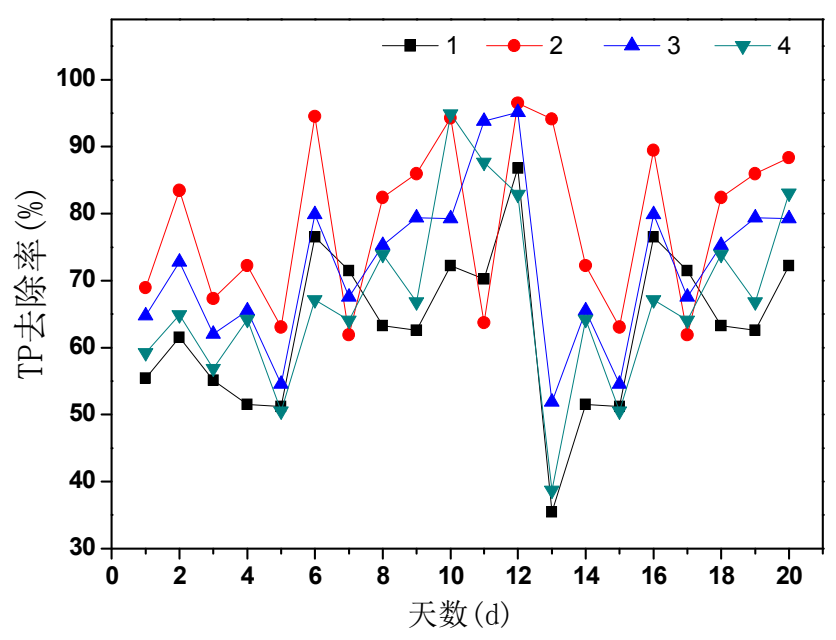

图8 不同光照时间运行条件下总磷的去除情况。

\section{4. 不同光照时间下悬浮固体含量的变化}

光照时间的不同对各反应器中悬浮固体含量的影响 如图 9 所示, 四组反应器的 MLSS 平均浓度分别是 $1805 \mathrm{mg} / \mathrm{L} 、 2174 \mathrm{mg} / \mathrm{L} 、 2023 \mathrm{mg} / \mathrm{L}$ 和 $1993 \mathrm{mg} / \mathrm{L}$ 。由此可知, 四组反应器的悬浮固体含量虽较为接近, 但是 2 号反应器 中悬浮固体的含量最高, 且始终较为稳定。而 1 号则波动 过大, 且基本在 $2000 \mathrm{mg} / \mathrm{L}$ 以下。因此在本实验中污泥活 性保持较好。根据污泥沉降比 (SV) 的数据可以看出, 污泥均无较大程度的膨胀。藻类生长过程是悬浮状态, 菌 藻共生体没有发生污泥膨胀的情况就可以确定共生体可 以稳定地存在不会相互影响。

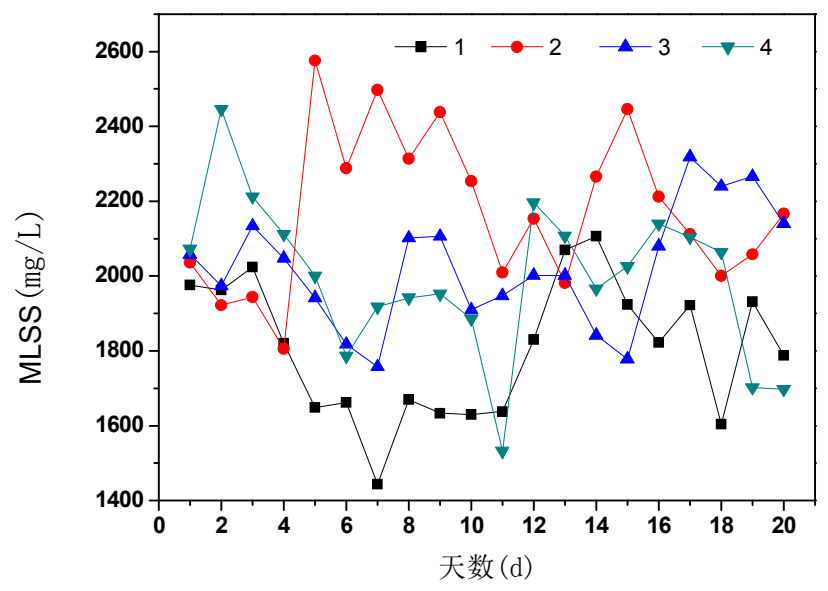

图9 不同光照时间运行条件下悬浮固体含量的变化。

\section{4. 结论}

对于菌藻共生SBR, 在一个完整的反应周期内, 光照 时间为 $8 \mathrm{~h}$, 黑暗状态为 4 小时, 即 2 号反应器的 COD去除效 果最为明显; 光照时间为 10 个小时的 3 号反应器次之。而 光照时间过长或者过短都不利于菌藻共生系统对 COD的 去除 $[11]$ 。应将菌藻SBR的光照时间定为 8 小时。

四组反应器对于氨氮的去除效果均在 $85 \%$ 以上, 其中, 3 号反应器的去除率最高, 2 号次之。由此可见, 光照时间 为 8 小时或者 10 小时都有利于提高菌藻共生系统对氨氮的 去除, 且二者的去除效率较为接近, 从节约能源的角度来 讲, 选择光照时间为 8 小时更为适宜。对于总氮, 2 号反应 器的去除效果最佳。

光照时间对磷去除效果的影响也较大，不论是总磷 还是正磷酸根, 均是 2 号反应器的去除效果较为突出。说 明光照时间为 8 小时跟利于藻类的生长, 过长时间的光照 可能对藻类的生长繁殖起到了抑制作用，而过短时间的 光照导致藻类吸收光能不足, 从而降低了藻类的生长活 性。

对比四组反应器的悬浮固体浓度可知, 首先, 四组 反应的生物活性均较为良好, 没有出现过大的波动; 其 次, 第二组和第三组的MLSS最高, 而且在反应后期没有 出现污泥老化的现象。说明适宜的光照时间更利于菌藻 的结合与生长。四组反应器在整个反应周期内均保持了 良好的沉降性能。所以当光照时间为 8 小时, 最适合污泥 与藻类的生长。

\section{致谢}

感谢政府间国际科技创新合作重点专项中德合作项 目的资助（2016YFE0123400），同时感谢城市水资源与 水环境国家重点实验室自主课题（2017DX02）的支持。

\section{参考文献}

[1] 巫小丹, 阮榕生, 王辉等. 菌藻共生系统处理废水研究现 状及发展前景 $[J]$. 环境工程, 2014, 32(3): 34-37.

[2] YanyanSu, ArturMennerichBrigitteUrban. Comparison of nutrient removal capacity and biomass settleability of four high-potential microalgalspecies[J]. Bioresource Technology, 2012, (124): 157-162.

[3] SofieVan, DenHende, ErwanCarréElodieCocaud, VeerleBeelen, NicoBoon, HanVervaeren. Treatment of industrial wastewaters by microalgal bacterial flocsin sequencing batch reactors[J]. Bioresource Technology, 2014, (161): 245-254.

[4] Sofie Van, Den Hende, Erwan Carré, Elodie Cocaud, Veerle Beelen, Nico Boon, Han Vervaeren. Treatment of industrial wastewaters by microalgal bacterial flocsin sequencing batch reactors[J]. Bioresource Technology, 2014, (161): 245-254. 
[5] N. C. Boeleea, H. Temmink, M. Janssen, C. J. N. Buisman, R. $\mathrm{H}$. Wijffels. Balancing the organic load and light supply in symbiotic microalgal-bacterial biofilm reactors treating synthetic municipal wastewater[J]. Ecological Engineering, 2014, (64): 213-221.

[6] 乔春, 汤金如, 沈希光. SBR 工艺污水处理技术 $[J]$. 安 阳学院学报, 2009(4): 44-47.

[7] 王凯军, 宋英豪. SBR工艺的发展类型及其应用特性 $[\mathrm{J}]$. 中国给水排水, 2002, 18(7): 23-26.

[8] And S M, Mataalvarez J. Utilization of SBR Technology for Wastewater Treatment: An Overview[J]. Ind.eng.chem.res, 2002, 41(23): 5539-5553.
[9] Julia M Valigore, Peter A Gostomski, David G. et al. Effects of hydraulic and solids retention times on productivity and settleability of microbial (microalgal-bacterial) biomass grown on primary treated waste-water as a biofuel feedstock[J]. water research, 2012, 46: 2957-2964.

[10] XU Meng, LI Ping, TANG Tianyu. Roles of SRT and HRT of an algal membrane bio-reactor system with a tanks-in-series configuration for secondary wastewater effluent polishing $[\mathrm{J}]$. Ecological Engineering, 2015, (85): 257-264.

[11] 潘辉, 熊振湖, 金勇威. 光照对固定化菌藻反应器脱氮除 磷效率的影响 [J]. 水资源保护，2006, 22(5): 63-64. 